\title{
BLOCKING T-CELL NEGATIVE SIGNAL REDUCE TUMOR BURDEN TO MINIMAL
}

\author{
T. K. GosWAMI \\ Immunology Section \\ Indian Veterinary Research Institute \\ Izatnagar-243 122, U.P., India
}

\begin{abstract}
Sustainable T-cell activation for tumor regression without inducing autoimmunity has been targeted by the cancer biologist. Alternatively blocking the T-cell negative signaling that has a retarding effect on T-cell activation signal may preclude tumor progression an unusual approach has been proposed recently. The proponent of the new concept James Patrick Allison working at US and Tasuku Honjo from Japan conceptualized that activation signal is similar like accelerator and negative signal is brake of a moving car, therefore releasing the brake may propel the vehicle forward without additional acceleration. Both Allison and Honjo has shared the Nobel Prize in Physiology and Medicine for the year 2018 for their original concept of blocking T-cell negative signal in cancer immunotherapy. Specific blocking of CTLA-4 and PD-1 two distinct protein molecules expressed on T-cell by Allison and Tasuku Honjo respectively using monoclonal antibody eventually leads to tumor regression in clinical cases.
\end{abstract}

Key words: Cancer, CTLA-4, Nobel Prize, PD-1, T-cell

Whether it is in man or animal invariably cancer is considered to be fatal. Cancer is very common in dogs and cats. For veterinarians diagnosis and treatment of cancer in present situation should acquire recent information. Cancer treatment involving radiotherapy to surgery even chemotherapy to complementary therapy are not new but success is few.
Immunotherapy is the concept of recent and over the world scientific community has given their consent. Using immune check point inhibitors to block the T-cell mediated negative signaling can augment the tumor regression has been proposed by the Nobel Prize Winner in Medicine and Physiology for the year 2018. It is similar like that of brake releasing phenomena in a moving 
vehicle, so that $\mathrm{T}$-cell activation signals progress smoothly without hindrance from negative signal. James Patrick Allison and Tasuku Honjo the Nobel Prize winner described that $\mathrm{T}$-cell activation signal as accelerators and inhibitory signal as brake in immune system. Both accelerator and brake act in a coordinate manner to remove the foreign invaders and maintain the harmony avoiding exaggerated response like auto-immunity. This concept was adapted with different approach to act against tumor cell sparing the self.

\section{Self non-self concept in immune system}

Discrimination of self from non-self is the criticality of vertebrate immune system. To tolerate self and to attack non-self is learnt by the developing embryo of vertebrates, so that invading bacteria, virus and other potential danger molecules are attacked for their elimination. T-cells can recognize any thing non-self whether it is outsiders like bacteria or virus or even insiders like own cells once self behave like non-self. Abnormal cells like cancer cells exhibit membrane expressed protein that differs from the normal healthy cell. Each T-cell receptors bind to specific molecules recognized as non-self on its target. Receptor target interaction triggers the immune system for activation. Once a Tcell receptor encounter any abnormal protein on target surface the activation signal of T-cell is switched on simultaneously another co-stimulatory signal also kick-starts engaging different receptors with corresponding ligand to drive the activation signal to progress without termination. This is known as two signal model of $\mathrm{T}$-cell activation. Besides these two signals, another negative signal also operates in parallel so that the end result is moderate and remains in progress rather than excess.

\section{Immune check point inhibitors}

Membrane expressed T-cell receptors are immensely variable unlike that of any other receptors present on it. These receptors are specifically designed to bind with a particular defined antigenic epitope. During circulation these $\mathrm{T}$-cell react against foreign cells or self cells expressing abnormality on its membrane that may be either altered self or missing self protein as the case may be. The cancer cells are considered to be own cell exhibiting abnormality, therefore become the target of T-cell attack. The event of encounter of T-cell with tumor leads to binding of TCR with the newly expressed antigenic epitope on tumor surface as well as binding of other accessory molecule of T-cells with its target. Binding of accessory molecules of T-cell with its target supports sustainable adherence therefore activation signal become operational. This is known as two signal model activation. Membrane expressed protein on T-cell named as CD28 binds with specific group of protein under B7 family i.e. CD80/CD86 that initiates costimulation. Role of B7 molecule for co- 
stimulation was demonstrated by transfecting $B 7$ gene in tumor cell caused early tumor regression (Linsley et al., 1992.; Townsend and Allison, 1993). CD28 proteins are constitutively expressed on $\mathrm{T}$ cells. Brunet and his group reported another protein known as CTLA-4 (cytotoxic T lymphocyte associated protein 4) expressed on T-cell (Brunet et al., 1987). CTLA-4 can bind equally well with $\mathrm{B} 7$ protein family on target (CD80/CD86) like that of CD28. It also acts as co-stimulatory molecule. Unlike CD28 binding, CTLA-4 exerts a negative signal upon T-cell ultimately the activation signal initiated by CD28 is retarded. Therefore CD28 and CTLA-4 molecule function is known to be in opposite manner, former supports activation signal and the later contribute in negative signaling. Unlike CD28, which is constitutively expressed, CTLA-4 are not constitutively expressed rather its expression follows the activation signal. Inactivation of ctla-4 gene in mice confirmed its negative regulatory role. Monoclonal antibody against CTLA-4 may block the negative signal in T-cell costimulation path thereby drive the T-cell activation for tumor regression was attempted by James Allison and his group. In subsequent experiment mice transplanted with tumor were treated with monoclonal against CTLA-4 resulted extensive tumor rejection with durable immunity (Leach $e t$ al., 1996). Commercial production of monoclonal antibody against CTLA-4 protein named MDX-010 later marketed by Bristol Myers Squibb Company with trade name as ipilimumab once approved by FDA in 2011.

In 1992, scientists from the laboratory of Tasuku Honjo from Japan discovered a different protein PD-1 (program death-1) on T-cells surface without any known function of it (Ishida et al., 1992). Tasuku Honjo professor at Kyoto University reported that PD-1 also act as negative signal inducer for T-cell. Protein PD-1 bind with a distinct ligand on target identified as PDL-1 and PDL-2 (L stands for ligand) resulting inhibitory signal to down regulate T-cell activation. The ligand PDL-1/PDL2 neither binds with CD28 nor to CTLA-4 indicating its identity as distinct grouped under B7 family protein B7-H-1. Tumor cells expressing PDL1 and PDL-2 prevent T-cell mediated cytotoxicity and rescuing the tumor cells from immune attack was proposed (Dong et al., 2002; Iwai et al., 2002). Honjo conceptualized the potential of an antibody against PD-1 to block the ligand binding property of PD-1. The mice with experimental cancer were treated with anti PD-1 monoclonal antibody and result was excellent (Iwai et al., 2005). The findings justified for its clinical application and commercial production of antibody named pembrolizumab /nivolumab by Ono pharmaceutical came to market and got approval from FDA in 2015. Long term remission of cancer using antibody against CTLA-4 and PD-1 proteins from clinical findings has established the proof of concept. The idea of "cancer immunotherapy; breaking the barriers to 
harvest the crop" as described by scientific group may be a realistic approach for cancer treatment in future (Pardoll and Allison 2004). Clinical trial across the world is

\section{REFERENCES}

Brunet JF, Denizot F, Luciani MF, RouxDosseto M and Suzan, M et al., 1987. A new member of the immunoglobulin superfamily CTLA-4. Nature, 328(6127): 267-270

Dong H, Strome SE, Salomao DR, Tamura H and Hirano F et al., 2002. Tumorassociated $\mathrm{B} 7-\mathrm{H} 1$ promotes $\mathrm{T}-$-cell apoptosis: a potential mechanism of immune evasion. Nat Med, 8(8): 793800

Ishida Y, Agata Y, Shibahara K and Honjo T, 1992. Induced expression of PD 1, a novel member of the immunoglobulin gene superfamily, upon programmed cell death. EMBO J, 11(11): 38873895

Iwai Y, Ishida M, Tanaka Y, Okazaki T and Honjo T et al., 2002. Involvement of PD-L1 on tumor cells in the escape from host immune system and tumor immunotherapy by PD-L1 blockade. Proc Natl Acad Sci, 99(19): 1229312297 presently using monoclonal against CTLA4 and PD-1 to reduce the tumor burden in human society, and adding the quality life in cancer patient.

Iwai Y, Terawaki S and Honjo T, 2005. PD-1 blockade inhibits hematogenous spread of poorly immunogenic tumor cells by enhanced recruitment of effector T cells. Int Immunol, 17(2): 133-144

Leach DR, Krummel MF and Allison JP, 1996. Enhancement of antitumor immunity by CTLA-4 blockade. Science, 271(5256): 1734-1736

Linsley PS, Wallace PM, Johnson J, Gibson MG and Greene JL et al., 1992. Immunosuppression in vivo by a soluble form of the CTLA- 4 T cell activation molecule. Science, 257: 792-795

Pardoll D and Allison J, 2004. Cancer immunotherapy: breaking the barriers to harvest the crop. Nat Med, 10(9): 887

Townsend SE and Allison JP, 1993. Tumor rejection after direct co-stimulation of CD8+ T cells by B7-transfected melanoma cells. Science, 259(5093): 368-370

Article received on 22.10.2018 and accepted for publication on 09.11.2018 